\title{
Fabrication and Characterization of Laser Scribed Supercapacitor based on Polyimide for Energy Storage
}

\author{
TAYYABA Malik ${ }^{\mathrm{a}}$, SHAYAN Naveed ${ }^{\mathrm{b}}$, MUHAMMAD Munir ${ }^{\mathrm{c}}$, \\ MOHAMMAD Ali Mohammad ${ }^{\mathrm{d}, *}$
}

\author{
School of Chemical and Materials Engineering (SCME), National University of Sciences and \\ Technology (NUST), Sector H-12, Islamabad 44000, Pakistan \\ a tayyaba.malik16@gmail.com, ${ }^{b}$ shayan_nse@scme.nust.edu.pk, ${ }^{c}$ muneer@scme.nust.edu.pk \\ ddr.ali@scme.nust.edu.pk
}

\section{Keywords: Polyimide (PI), Reduced Polyimide (rPI), Supercapacitor, Flexible Electronics}

\begin{abstract}
Recently, supercapacitors have attracted a tremendous amount of attention as energystorage devices due to their high-power density, fast charge-discharge ability, excellent reversibility, and long cycling life. In this research work, we demonstrate a laser scribed super capacitor based on polyimide (PI) substrate for the storage of electrical energy. PI substrate of thickness $200 \mu \mathrm{m}$ and area $1 \mathrm{~cm} \times 1 \mathrm{~cm}$ was reduced by a laser engraver with a $450 \mathrm{~nm}$ wavelength in the form of stackable supercapacitor electrodes. Although, PI itself exhibits non-conductive behavior; however, by laser irradiation we change the surface properties of PI and reduce its resistance. The chemical property of irradiated PI was characterized with XRD where the carbon peak was observed at $2 *$ theta $=25.44$, which confirms the reduction of PI material in to a graphenelike substance. The electrical conductivity was analyzed with a probe station and observed to be $1.6 \mathrm{mS}$. Two conductive regions were assembled into a capacitor device by sandwiching a $\mathrm{PVA} / \mathrm{H}_{3} \mathrm{PO}_{4}$ electrolyte in between. During the charging and discharging characterization of the capacitor device, current density was observed to be $1.5 \mathrm{~mA} / \mathrm{cm}^{2}$. Capacitance versus voltage analysis was carried out and the device showed $75 \mathrm{mF} / \mathrm{cm}^{2}$ against a voltage sweep of $\pm 2 \mathrm{~V}$. The galvanostatic charging and discharging curve shows a symmetric behavior with respect to time exhibiting the stability and durability of the device.
\end{abstract}

\section{Introduction}

Energy generation and storage is a topic of great contemporary importance and hence efforts are being made across the globe to develop solutions in this domain. For energy storage, a wide range of devices have been proposed which vary in application area, storage capacity, retention, charge/discharge speed, form, size, etc. Besides batteries and electronic memory devices, supercapacitors are a very famous device for the energy storage [1]. It has the ability to store energy and it has high-power density and long cycling life [2]. Supercapacitors are expected to impact consumer electronics, electrical power grids, transport and military applications, and various types of electrical instruments for commercial and industrial uses. Supercapacitors are mostly formed of graphene and graphene like material, which have yielded favorable results in terms of power density and long cycling life. Graphene based materials have a porous three dimensional structure with a large surface area due to which it has the ability to store energy and hence highly desirable for supercapacitors[3]. These supercapacitors not only shows high surface area but also shows good electrical conductivity which is very important parameter for large energy and power. They can be used in flexible electronics devices. Graphene based supercapacitors are very beneficial but graphene is expensive and its synthesis process is complex [4]. For graphene synthesis many chemical steps are required and on commercial scale its yield is not very high. Other than graphene based supercapacitors, direct laser writing on graphite oxide [5, 6], light scribed DVD drive [7] and $\mathrm{CO}_{2}$ laser based techniques are used to fabricate the supercapacitors[8]. In these techniques, graphene oxide is converted in to the reduced graphene oxide using laser beams which results in a porous structure with a surface amenable for electron transport and charge storage. Laser induced 
graphene by laser scribing is a well-known method for the fabrication of supercapacitor electrodes [9]. In the literature it is shown that the capacitance is dependent on its physical geometry and substrate materials $[10,11]$. The performance of supercapacitor is also dependent on the intensity of laser power during fabrication, which should be investigated.

In this paper we use a single step and low cost method of producing a carbonized structure by using laser technology. A small laser setup is used to change the surface of polyimide. It is an insulating polymer and has non-conductive properties. Upon irradiation by a laser, its surface is converted into carbonized material which has high porosity. Now this reduced polyimide (rPI) has high conductive properties as compared to a simple polyimide sheet. Having high density, porous structure, and good conductivity, rPI is used for supercapacitor applications. By using this simple laser set up flexible supercapacitor of different types can be formed. Here we checked the behavior and capacitance of our device by changing the design, size, scan rate and laser intensity. In addition, previously silver paste and copper type was used for electrode contacts due to which some kind of resistance existed and to avoid a loss of performance due to this resistance, in this paper we have made some changes in the design. The electrodes are also formed by the same material (reduced polyimide), due to which there is no change or loss of conductivity. These kinds of supercapacitors are flexible, smaller and cheaper in price for consumer purpose. For its design there is no need of any lithography technique, and any kind of design can be imported into the software. By using its simple software, the laser intensity and carving time can be varied. The intensity used in this paper is $111 \mathrm{~mW}$ and $139 \mathrm{~mW}$. At a higher intensity we get better electrochemical results.

\section{Experimentation}

Fabrication of Supercapacitor. The fabrication of the supercapacitors was performed by using commercial polyimide of $200 \mu \mathrm{m}$ thickness. A laser engraver of $450 \mathrm{~nm}$ wavelength and maximum power $500 \mathrm{~mW}$ [12] was used to reduce the surface of PI and convert the irradiated area in to a carbonized structure. Fig. 1(a) shows a photograph of the instrument. It is a 2D laser setup in which the laser moves in the X-Y direction by using stepper motors. This instrument does not require any mask for the fabrication and arbitrary designs can be drawn by importing a customized structure into the control software. By changing the laser intensity different types of stacked supercapacitors were formed with differing characteristics. In stack type supercapacitor, an area of $1 \mathrm{~cm} \times 1 \mathrm{~cm}$ of PI was reduced by the laser which converts it into the carbonized material having a porous structure. An additional elongated area, measuring $0.2 \mathrm{~cm} \times 1 \mathrm{~cm}$, was reduced besides the $1 \mathrm{~cm} \times 1 \mathrm{~cm}$ square which acts as an electrode to connect to external measurement apparatus. A polymeric electrolyte $\mathrm{PVA} / \mathrm{H}_{3} \mathrm{PO}_{4}$ is sandwiched in between two $1 \mathrm{~cm} \times 1 \mathrm{~cm}$ areas and laminated to form a packaged prototype (schematically shown in Fig. 1(b)). The polymeric electrolyte $\mathrm{PVA} / \mathrm{H}_{3} \mathrm{PO}_{4}$ was made by mixing and stirring $10 \mathrm{ml}$ of DI water, $1 \mathrm{ml}$ of $\mathrm{H}_{3} \mathrm{PO}_{4}$ and $1 \mathrm{~g}$ PVA and heated at $80^{\circ} \mathrm{C}$ overnight [12, 13]. As a result, a gel type electrolyte is formed, which is coated on rPI and then placed in an oven at $50^{\circ} \mathrm{C}$ for 45 minutes to remove the excess water present in the electrolyte.

\section{Characterization}

Electrochemical testing of the assembled supercapacitor device was done by using an ECI workstation and EC-lab software. The conductivity test of rPI electrodes was performed using a two-point probe station. X-Ray Diffraction (XRD) data for PI and rPI was obtained using a STEO theta-theta diffractometer. Similarly, Fourier Transform Infrared Spectroscopy (FT-IR) data for PI and rPI was obtained using a BRUKER Alpha. High resolution SEM images of various rPI regions were obtained using a JEOL Analytical SEM (JSM-6490A). 

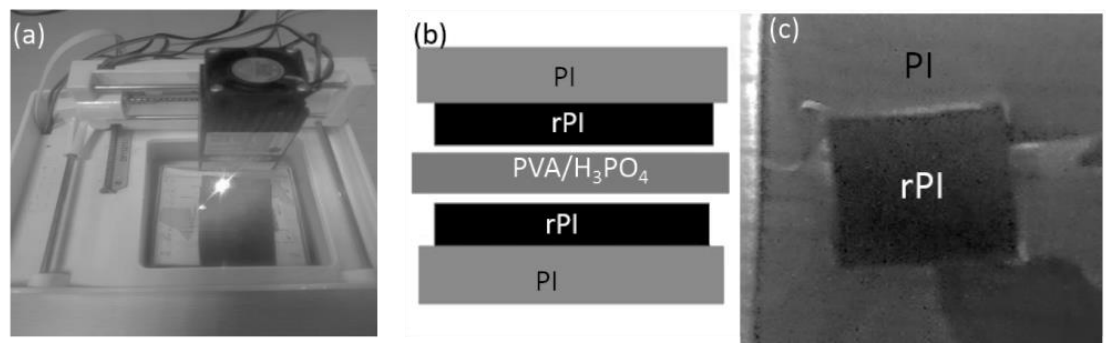

Fig. 1. (a) A digital image of a diode laser setup having a maximum power of $500 \mathrm{~mW}$ and $450 \mathrm{~nm}$ wavelength, (b) a schematic diagram of the stacked supercapacitor, and (c) an optical photograph of the fabricated single electrode.

\section{Results and Discussion}

The reduced polyimide electrodes were characterized by optical microscopy, SEM, XRD, FT-IR, and electrical two-point probing. Fig.1 (c) shows an optical micrograph of a rPI electrode after laser irradiation. The surface is converted from a brownish solid form into a black porous form which resembles a carbonized area. Fig. 2(a) shows a SEM image of the reduced PI by laser scribing of PI substrate in which it is clearly shown that the surface morphology of PI gets changed after the laser irradiation. A zoomed image of reduced PI in Fig. 2(b) shows the flakes and porous carbon like material which have higher surface density and interconnections with each other. This porous surface look like a honeycomb structure that is very useful for storage purposes. The roughened surface with interconnected pores enables excellent electrolyte contact and contributes towards a high capacitance value. Fig. 2(c) shows the cross sectional view of reduced PI on PI substrate which shows that the structure increases in thickness following laser irradiation.

PI is a non-conductive polymer and when it is reduced by laser irradiation, it becomes conductive [15]. Fig. 2(d) shows the conductivity graph in which it is shown that by using a two probe station with a $0-2 \mathrm{~V}$ sweep, PI shows a maximum current output of $0.1 \mathrm{pA}$, whereas rPI shows a maximum current output of $3.2 \mathrm{~mA}$ and a linear graph. The relative magnitude of current output and the linear graph shows the conversion of insulating PI material into conductive rPI material. By using Ohm's law, we find the value of resistance and subsequently calculate the approximate conductance value which is equal to $1.6 \mathrm{mS}$.
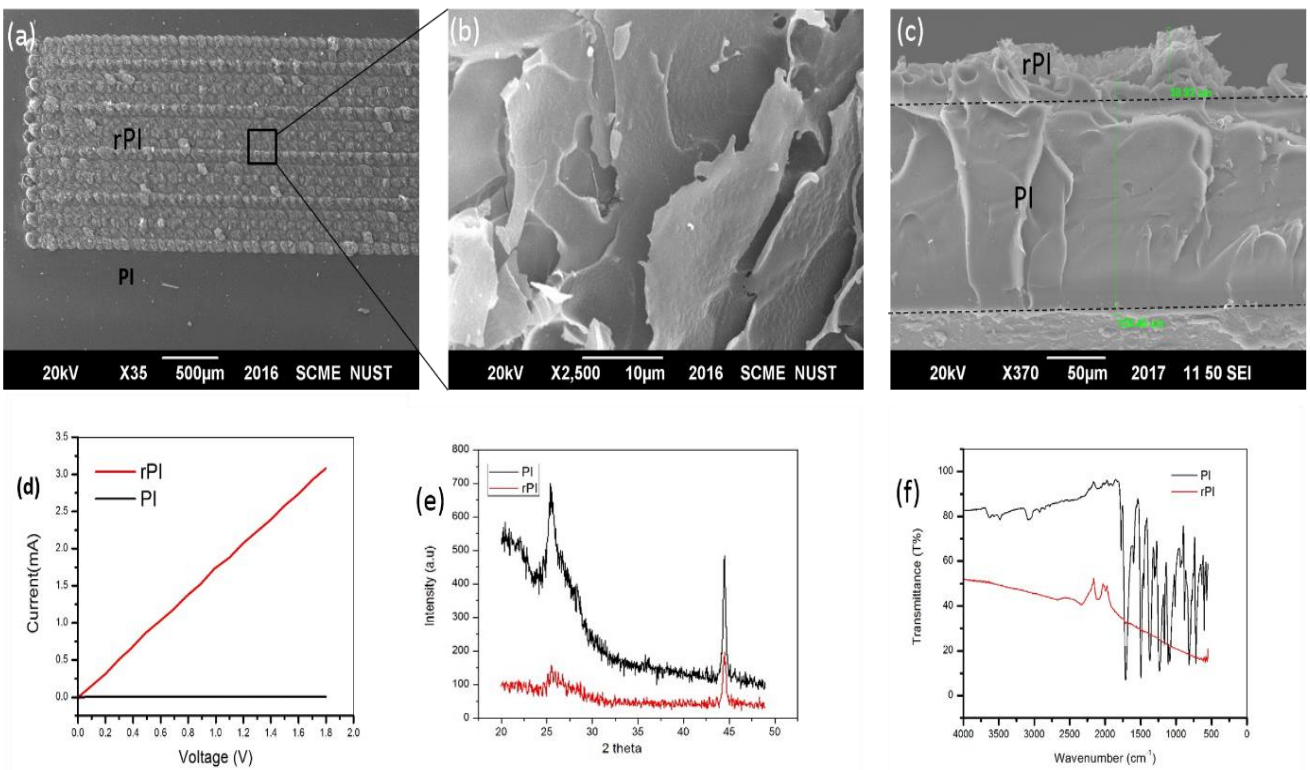

Fig. 2. (a) SEM image of the reduced PI by laser scribing on PI substrate, (b) Zoomed SEM image of the reduced area of PI, (c) cross section SEM image of the reduced PI, (d) conductivity test of PI and reduced PI, (e) XRD image of PI and reduced PI, and (f) FTIR spectra of PI and reduced PI. 
XRD patterns of PI and rPI material is shown in Fig. 2 (e). PI substrate shows a very high intensity peak at $2 \theta=25.44$ and after laser irradiation the reduced PI material shows a small low intensity peak at $2 \theta=25.44$ which indicates the reduction into the graphene like material. In Fig. 2 (f) FTIR spectra of PI shows peaks from $620-1779 \mathrm{~cm}^{-1}$ and this matches with commercial polyimide FTIR results having stretching and bending modes of the $\mathrm{C}-\mathrm{O}, \mathrm{C}-\mathrm{N}$ and $\mathrm{C}=\mathrm{C}$ bonds [9]. FTIR spectra of laser reduced PI shows a large change in its peaks from $620-1780 \mathrm{~cm}^{-1}$. These changes indicate that the bond structure has changed in the region of interest and especially the disruption of $\mathrm{C}-\mathrm{O}$ structure indicates reduction.

Fig. 3 shows the electrochemical testing results that were conducted using electrochemical lab setup. Cyclic Voltammetry and charge discharge measurements was taken for the supercapacitor devices made at different laser intensities. Cyclic voltammetry measurements were taken on different scan rates. By changing the scan rate the capacitance value also changes. There are two parameters which we varied and on which we checked the supercapacitor value. The first one is the intensity of the laser, and the second one is the scan rate.
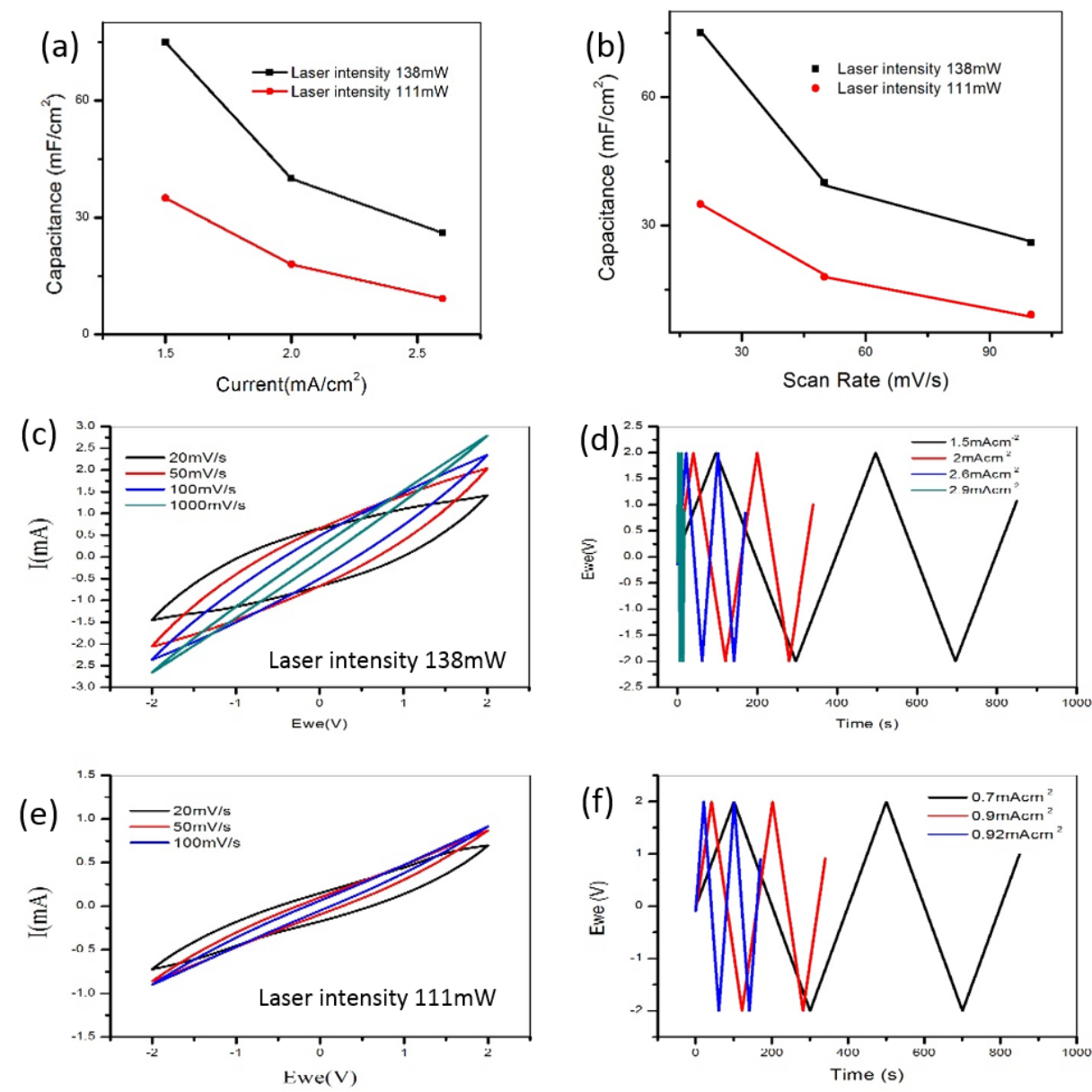

Fig. 3. (a) Capacitance vs current analysis of the proposed supercapacitor, (b) Capacitance vs. scan rate for two different laser intensities, (c) Current vs voltage sweep at $138 \mathrm{~mW}$ intensity and at various scan rates, (d) charging and discharging cycles analysis of the device fabricated at $138 \mathrm{~mW}$ laser intensity, (e) Current vs. voltage sweep at $111 \mathrm{~mW}$ intensity and at various scan rates, and (f) charging and discharging cycles analysis of the device fabricated at $111 \mathrm{~mW}$ laser intensity.

Laser reduced PI capacitor exhibits a maximum capacitance value of $75 \mathrm{mF} / \mathrm{cm}^{2}$ at the intensity value of $138 \mathrm{~mW}$ and a minimum value of $35 \mathrm{mF} / \mathrm{cm}^{2}$ at an intensity of $111 \mathrm{~mW}$. CV results shows that by increasing the scan rate the capacitance values decrease and by increasing the laser intensity to an optimum value we get better capacitance value as shown in Fig. 3(a,b). By using equation (1) and (2) [16] we calculate the capacitance value, where I is the average current, A is the surface area of electrode which is $1 \mathrm{~cm}^{2}$ and $\mathrm{dv} / \mathrm{dt}$ is the scan rate in $\mathrm{mV} / \mathrm{s}$. 


$$
\begin{aligned}
& C_{\text {device }}=\frac{\mathrm{I}}{\frac{\mathrm{dV}}{\mathrm{dt}}} \\
& C_{\text {areal }}=\frac{C_{\text {device }}}{A}
\end{aligned}
$$

There is another type of equation used in this paper for the calculation of capacitance. Using equation (3), the capacitor value is calculated from the CV curve. Here $\mathrm{I}(\mathrm{V})$ is the total current enclosed in the CV curve in $\mathrm{mA}, \mathrm{S}$ is the specific active area of electrodes which is $1 \mathrm{~cm}^{2}, \mathrm{dV} / \mathrm{dt}$ is the scan rate in $\mathrm{mV} / \mathrm{s}$ and $\mathrm{V}_{\mathrm{f}}$ and $\mathrm{V}_{\mathrm{i}}$ are the voltage limits.

$$
C_{A}=\frac{1}{2 \times S \times\left(v_{f}-v_{i}\right) \times \frac{d V}{d t}} \int_{v_{i}}^{v_{f}} I(V) d V
$$

These supercapacitors show excellent cyclic stability; the capacitance remains unchanged after 100 cycles of charge/discharge at a current density of $2.6 \mathrm{~mA} / \mathrm{cm}^{2}$ and at a scan rate of $100 \mathrm{mV} / \mathrm{cm}^{2}$. Galvanostatic charge and discharge cycles are shown in Fig. 3(d,f) at different current values which displays symmetric triangular plots throughout with increasing time.

\section{Conclusions}

In this work, we have reported the fabrication and characterization of a supercapacitor which uses laser reduced polyimide as the electrodes. Laser reduction is a low cost and relatively straightforward method to reduce PI and convert a surface layer into a carbonized material having high porosity. Reduced PI has a high storage capacity and we have analyzed the performance of our $\mathrm{rPI} /\left(\mathrm{PVA}+\mathrm{H}_{3} \mathrm{PO}_{4}\right) / \mathrm{rPI}$ supercapacitor by varying the laser intensity from $83 \mathrm{~mW}$ to $167 \mathrm{~mW}$. We found that $138 \mathrm{~mW}$ is the optimum power for the stacked supercapacitor geometry on a $200 \mu \mathrm{m}$ thick PI substrate and a maximum capacitance value of $75 \mathrm{mF} / \mathrm{cm}^{2}$ was obtained. From this we concluded that by changing the intensity of the laser to an optimum condition one can change the capacitance value. Further, by increasing the laser intensity, the capacitance value increased. At different scan rates, the capacitance value is different and increasing the scan rate decreases the capacitance value.

\section{Acknowledgements}

We would like to acknowledge funding from the Higher Education Commission (HEC) of Pakistan ref. grant no. 6040/Federal/NRPU/R\&D/HEC/2016 and grant no. HEC/R\&D/PAKUS/2017/783. We are also grateful to a number of Professors, such as Dr. Syed Rizwan Hussain (NUST) and Dr. Aftab Akram (NUST) for useful discussions and assistance with the ECI instrument, and Prof. Aslam Baig (NCP) for measurement of laser parameters. Special mentions for Lab Tech. Shams Uddin, Lab Tech. Khurram Shahzad, Lab Tech. Hafiz Moazzam Riaz for assistance with SEM, XRD, and FT-IR, respectively. Finally, we would also like to mention the Advanced Electronics Laboratory Pakistan (AELP) at the International Islamic University (IIUI) of Pakistan for access to their facilities and useful discussions.

\section{References}

[1] M. Beidaghi and C. Wang, Micro-Supercapacitors Based on Interdigital Electrodes of Reduced Graphene Oxide and Carbon Nanotube Composites with Ultrahigh Power Handling Performance, Adv. Funct. Mat., 222012 4501-4510.

[2] D. Pech, M. Brunet, H. Durou, P. Huang, V. Mochalin, Y. Gogotsi, et al., Ultrahigh-power micrometre-sized supercapacitors based on onion-like carbon, Nat. Nanotech. 5 2010 651-654.

[3] Y. Zhu, S. Murali, M. D. Stoller, K. Ganesh, W. Cai, P. J. Ferreira, et al., Carbon-based supercapacitors produced by activation of graphene, Sci., 3322011 1537-1541.

[4] D. C. Marcano, D. V. Kosynkin, J. M. Berlin, A. Sinitskii, Z. Sun, A. Slesarev, et al., Improved Synthesis of Graphene Oxide, ACS Nano, 42010 4806-4814. 
[5] W. Gao, N. Singh, L. Song, Z. Liu, A. L. M. Reddy, L. Ci, et al., Direct laser writing of microsupercapacitors on hydrated graphite oxide films, Nat. Nanotech. 62011 496-500.

[6] M. F. El-Kady, V. Strong, S. Dubin, and R. B. Kaner, Laser scribing of high-performance and flexible graphene-based electrochemical capacitors, Scie., 3352012 1326-1330.

[7] M. F. El-Kady and R. B. Kaner, Scalable fabrication of high-power graphene microsupercapacitors for flexible and on-chip energy storage, Nat. Commun., 420131475.

[8] Z. Peng, J. Lin, R. Ye, E. L. Samuel, and J. M. Tour, Flexible and stackable laser-induced graphene supercapacitors, ACS Appl. Mat. Interf., 72015 3414-3419.

[9] J. Lin, Z. Peng, Y. Liu, F. Ruiz-Zepeda, R. Ye, E. L. Samuel, et al., Laser-induced porous graphene films from commercial polymers, Nat. Commun., 520145714.

[10] J. B. In, B. Hsia, J.-H. Yoo, S. Hyun, C. Carraro, R. Maboudian, et al., Facile fabrication of flexible all solid-state micro-supercapacitor by direct laser writing of porous carbon in polyimide, Carb., 832015 144-151.

[11] L. Jiao and R. Gorby, Laser scribed graphene for energy storage application, in OptoElectronics and Communications Conference, 2015, pp. 1-3.

[12] L. Q. Tao, H. Tian, Y. Liu, Z. Y. Ju, Y. Pang, Y. Q. Chen, et al., An intelligent artificial throat with sound-sensing ability based on laser induced graphene, Nat. Commun., 8201714579.

[13] M. Kaempgen, C. K. Chan, J. Ma, Y. Cui, and G. Gruner, Printable thin film supercapacitors using single-walled carbon nanotubes, Nano lett., 92009 1872-1876.

[14] Q. Chen, X. Li, X. Zang, Y. Cao, Y. He, P. Li, et al., Effect of different gel electrolytes on graphene-based solid-state supercapacitors, RSC Adva., 42014 36253-36256.

[15] M. Inagaki, N. Ohta, and Y. Hishiyama, Aromatic polyimides as carbon precursors, Carb., 61 $20131-21$.

[16] J. Lin, C. Zhang, Z. Yan, Y. Zhu, Z. Peng, R. H. Hauge, et al., 3-dimensional graphene carbon nanotube carpet-based microsupercapacitors with high electrochemical performance, Nano lett., 13 $201272-78$. 